\title{
Nanoparticles in sentinel lymph node mapping
}

\author{
Gregory Ravizzini ${ }^{1}$, Baris Turkbey ${ }^{1}$, Tristan Barrett ${ }^{1}$, Hisataka Kobayashi ${ }^{1}$, and Peter L. \\ Choyke $^{1, *}$ \\ ${ }^{1}$ Molecular Imaging Program, National Cancer Institute, National Institutes of Health, Bethesda, \\ MD 20892, USA
}

\begin{abstract}
The lymph nodes and lymphatic vessels are more difficult to access than most vascular structures. Interstitial injection of imaging agents is often necessary to opacify the lymphatics. Traditionally, radionuclide methods of sentinel node imaging have dominated this field, however, limitations in resolution and exposure to radiation have encouraged the development of newer imaging methods. Among these are magnetic resonance lymphography in which a Gadolinium labeled nanoparticle is injected and imaged providing superior anatomic resolution and assessment of lymphatic dynamics. Optical imaging employing various nanoparticles including quantum dots also provide the capability of mapping each lymphatic basin in another "color". Taken together this "toolbox" of lymphatic imaging agents is poised to improve our understanding of the lymphatic system.
\end{abstract}

\section{INTRODUCTION}

Sentinel lymph node (SLN) imaging with nanoparticles could be a useful application of nanotechnology as the size range of $1-100 \mathrm{~nm}$ is well suited to uptake by the lymphatics. ${ }^{1}$ Traditional SLN imaging with radiolabeled and blue dye nanoparticles is well established in the clinical arena, but research suggests that whole families of new nanoparticles could be used for SLN imaging. Here, we present a brief review of the current developments in SLN imaging utilizing novel nanoparticles tagged with imaging beacons capable of detection using cameras made for nuclear medicine, computed tomography (CT), ultrasound (US), optical imaging, and magnetic resonance imaging (MRI). Table 1 gives examples of nanoparticles studied in SLN mapping.

\section{BACKGROUND}

Cancer spreads in a variety of ways: by direct extension, via the vascular system and via the lymphatics/lymph nodes. The presence of lymph node metastases is an important prognostic implication in most cancer types; ${ }^{2}$ besides modifying the stage of the disease, it is a major criterion for determining the need for adjuvant chemotherapy. ${ }^{3}$ The SLN is the first lymph node that drains the primary tumor. ${ }^{4}$ Echelon lymph nodes are unlikely to be affected if the SLN is normal whereas, they may be involved if the SLN contains cancer cells. Thus, there is a generally orderly progression of cancer from the primary tumor to the SLN and then on

Correspondence to: pchoyke@nih.gov. 
to echelon lymph nodes. ${ }^{5}$ SLN localization is particularly important for the treatment of melanoma, breast, cervical, and head and neck cancers where the status of the SLN is frequently used as part of surgical management. By harvesting only the SLN and any immediately adjacent nodes, determining their tumor status and performing more aggressive surgery only in selected patients, an overall reduction in surgical morbidity, specifically extremity lymphedema, can be achieved. ${ }^{6}$

For the past few decades, noninvasive imaging modalities such as CT, MRI, and positron emission tomography have been used to identify abnormally enlarged lymph nodes which suggest the presence of cancer. ${ }^{7}$ These techniques, while appealing, are of limited value because they cannot determine which node is the SLN and also are unreliable in establishing the presence of metastatic lymph nodes as the diagnostic criterion is on the basis of node size; small nodes, below the size threshold, can nonetheless harbor metastases while large nodes may be hyperplastic because of chronic inflammation. ${ }^{8}$ Thus, despite dramatic improvements in the quality of noninvasive imaging, new methods directed at specifically identifying the SLN have been developed.

The basis for SLN imaging is that a nanoparticle injected near the primary tumor will be taken up by the adjacent lymphatics and transported to the SLN. Currently, there are two imaging agents for localizing SLNs in the United States; isosulfan or 'blue dye' (Lymphazurin 1\%, US Surgical, North Haven, CT) and radionuclide-labeled sulfur colloid. Variants of these agents are used in other countries. Isosulfan blue dye is injected into the peritumoral region and the SLN turns blue. This technique pre-supposes that the general region of the SLN is known and the surgeon dissects the skin to the expected general area of the SLN, honing in on the SLN when it is possible to directly visualize it. In the radionuclide technique also known as 'lymphoscintigraphy' (LS), ${ }^{99 \mathrm{~m}} \mathrm{Tc}$-labeled sulfur colloid is injected in the peritumoral or periareolar area and the SLN is located by using a handheld gamma ray counter. ${ }^{9}$ Both isosulfan blue and radiolabeled sulfur colloid can be used together to identify the SLN, the latter providing overall guidance on the location of the SLN and the former providing more specific localization. Isosulfan blue is approximately $20 \mathrm{~nm}$ in diameter and sulfur colloid can be filtered so that the particles are less than $220 \mathrm{~nm}$ in diameter. ${ }^{10,11}$

However, neither agent is ideal; peritumorally injected blue dye transits from the interstitial space through the lymphatic channels to the SLN; however, because the blue stain can only be detected for a few millimeters, the surgeon must accurately dissect down to the right location or risk missing the SLN altogether. Failure to identify the SLN will result in more extensive dissection defeating the purpose of the SLN procedure. ${ }^{12}$ Blue dye may cause a long lasting stain over the injected tissues so that in the case of breast SLN imaging, an unsightly stain on the breast can remain for months after the procedure. In addition, a 1.6\% rate of allergic reactions has been reported. ${ }^{13}$ With radionuclide labeled sulfur colloid, SLN imaging can detect SLNs deep to the skin and help localize the SLN; however, it has low spatial resolution, requires skill and training in the use of the handheld gamma counter and exposes both the patient and particularly the surgical team to ionizing radiation (the latter will be repeatedly exposed). Because both the injection site and the SLN will emit gamma rays, care must be taken to avoid falsely identifying the injection site as the SLN when using the handheld counter, a skill that must be developed over time. Another pitfall is that a 
grossly metastatic lymph node may cause blockage of the lymph flow through the afferent lymphatic channels preventing its identification during the procedure. ${ }^{14}$

\section{Sentinel Lymph Node Biopsy}

Sentinel lymph node biopsy (SLNB) is a relatively new, minimally invasive procedure which is gaining popularity for accurate determination of nodal status with decreased morbidity. It has been studied in the management of patients with breast cancer, melanoma, head and neck tumors, Merkell cell carcinoma, penile carcinoma, and gynecological malignancies. ${ }^{15-18}$ In this article, we will focus on SLNB application mainly in regard to breast cancer and melanoma.

The basic principle for SLNB was observed in 1907 when Jamieson described the significance of neoplastic cells spreading to the so-called 'primary gland' by which he meant the draining lymph node. ${ }^{19}$ The term 'sentinel node' - the first lymph node in a regional lymphatic basin, which receives flow from a primary tumor-was proposed by Gould et al. in 1960 when studying cancers of the parotid gland. In 1977, Cabanas ${ }^{5}$ demonstrated the feasibility of lymphatic mapping for the identification of SLNs in patients with penile carcinoma. However, the procedure gained momentum only in the early 1990s when Morton described the technique of lymphatic mapping in primary cutaneous melanoma using isosulfan blue dye. Intradermal injections were performed circumferentially around the primary lesion and the blue-stained lymphatics were followed surgically until a blue-stained lymph node was identified. In 1993, Alex and Krag, reported the first gamma-probe localization of SLNs utilizing ${ }^{99} \mathrm{~m}$ Tc-sulfur colloid in the animal model. They found good correlation in the identification of SLNs using gamma-probe guided LS and the isosulfan blue dye procedure. ${ }^{20}$ Subsequently, the same group described the application of this technique in the evaluation of patients with breast cancer. ${ }^{21}$ In this pilot study, axillary SLNs were identified in 18 out of 22 patients. In 1994, Giuliano and colleagues extended Morton's technique to breast cancer, injecting isosulfan blue dye into breast masses and the surrounding breast parenchyma. ${ }^{22}$ In 1996, Albertini performed a prospective trial where participants underwent intraoperative lymphatic mapping using a combination of a vital blue dye and ${ }^{99 \mathrm{~m}} \mathrm{Tc}$-sulfur colloid to improve diagnostic accuracy. ${ }^{23}$

${ }^{99} \mathrm{~m}$ Tc-sulfur colloid is approved for imaging of the liver and spleen by the United States Food and Drug Administration and is widely used for LS in the filtered and unfiltered forms. The smaller filtered particles are micro-filtered (pore size $220 \mathrm{~nm}$ Millipore, Bedford, MA). Unfiltered sulfur-colloid has variable range of sizes because of aggregation and can range from 100 to $1000 \mathrm{~nm}$ depending on the preparation technique. ${ }^{24}$ Particle size determines the kinetics of the lymphatic uptake of the radiotracer. ${ }^{25}$ Filtered particles migrate faster and have been noted to delineate a greater number of echelon lymph nodes. Worldwide, additional radiotracers are utilized. For example, ${ }^{99 \mathrm{~m}} \mathrm{Tc}$-antimony trisulfide (particle size, $15-300 \mathrm{~nm}$ ) is commercially available in Australia and Canada and ${ }^{99 \mathrm{~m}} \mathrm{Tc}-\mathrm{Human}$ Serum Albumin nanocolloid (particle size, $50-800 \mathrm{~nm}$ ) is used in Europe. ${ }^{26,27}$

Radiotracer dose, injection techniques and imaging protocols vary according to the type of tumor being investigated. For example, in patients with melanoma, dynamic or sequential imaging is usually performed immediately after the intradermal injection of radiotracer 
around the primary tumor and continued for $30-60$ min. ${ }^{28}$ After images are obtained, the patient is taken directly to the operating room. In breast cancer, the most popular approaches are to perform an intradermal injection in the skin overlying the primary tumor or subdermal injections in the periareolar area of the ipsilateral breast ${ }^{29,30}$ (Figures 1 and 2). Dynamic or sequential images are not routinely performed and images can be obtained on the same day or the day before surgery. ${ }^{31}$ For the 2-day protocol, unfiltered sulfur colloid is employed and because of the slower kinetics of the larger particle imaged later in the day. The patient is then operated on the following day. Comparable results are achieved with a 1 day protocol using filtered sulfur colloid in which the imaging and intraoperative localization are performed on the same day. Proponents of the 2-day protocol claim greater efficiency in scheduling with less operating room time wasted in waiting for patients to arrive from the Nuclear Medicine Department. ${ }^{31}$ The main disadvantage of LS is the poor spatial resolution and the lack of detailed anatomy to guide surgeons during excision. Recently, the introduction of new imaging instrumentation such as SPECT/CT promises more accurate depiction of lymphatic channels and draining lymph nodes. ${ }^{32}$ SPECT/CT will probably have its highest impact in tumors located in body parts with ambiguous lymph node drainage.

With the pre-operative images as a guide, the surgeon uses a small handheld probe to detect gamma-rays emissions from the radiotracer by means of auditory signals and meter read-out of counts (Figure 3). By placing the probe over the region of highest counts, an incision can be made directly over the SLN. A SLN is not necessarily the hottest node, although that is often the case. A SLN usually has at least 10 times the background counts, taken at a location remote from the injection site. ${ }^{28}$ After the SLN is removed, the probe can be used to detect residual activity and the presence of additional lymph nodes.

The ample use of screening mammography has resulted in women having smaller tumors at the time of initial presentation and a lower risk of involved lymph nodes. ${ }^{33}$ Wide local excision followed by external beam radiation therapy (EBRT) to the whole breast is rapidly becoming the standard of care for most patients with localized breast cancer in the developed world. ${ }^{34,35}$ The presence of axillary lymph node metastases represents the single most important prognostic indicator in patients diagnosed with breast cancer. It has been shown that the presence of regional metastases within the axillary basin decreases a patient's 5-year survival by approximately $28-40 \%{ }^{36,37}$ Axillary lymph nodes receive $85 \%$ of the lymphatic drainage from the breast; the remainder drains to the internal mammary chain. The likelihood of axillary lymph node $(\mathrm{LN})$ involvement is related to histologic grade of the primary tumor and the presence of lymphatic or vascular invasion in the specimen from the primary tumor. Low grade tumors (grade 1) have a lower likelihood of nodal spread than those with higher grade ( 2 or 3 ). ${ }^{38}$ Tumor size and location also play an important role. For example, there is a $16-19 \%$ chance for the presence of axillary node metastases in very early breast cancers (T1a-b, tumor size $\leq$ to $1 \mathrm{~cm}$ ). ${ }^{39} \mathrm{In} \mathrm{T} 1 \mathrm{c}$ lesions (size 1-2 cm), this increases to $30-40 \% .{ }^{40}$ Axillary lymph node metastasis is more common in patients in whom the primary tumor is located in the outer quadrants of the breast. ${ }^{41}$ Physical examination has poor predictive value for the determination of nodal involvement. Up to one-third of women with nonpalpable axillary lymph nodes will harbor metastases, while one-third of those with palpable lymph nodes will be disease free. Historically, axillary lymph node dissection (ALND) was used for staging and prevention of locoregional recurrence. However, the 
procedure produces considerable morbidity in the form of lymphedema and sensory motor disturbances in the ipsilateral extremity and is of unproven benefit in comparison to adjuvant chemotherapy which shows clear therapeutic benefit. For these reasons, the use of full ALND for staging purposes in clinically low risk patients is declining with the adoption of the less invasive SLNB technique. In 2006, Kim et al. published a meta-analysis of the World's literature of SLNB in patients with early-stage breast carcinoma. ${ }^{42}$ Sixty nine trials extending from 1970 to 2003 were included. Of the 8059 patients studied, 7765 patients (96\%) had successfully mapped SLNs. Lymph node involvement was found in 3132 patients (42\%) and ranged from 17 to $74 \%$ across studies. The false-negative rate (FNR) ranged from 0 to $29 \%$, averaging $7.3 \%$. Eleven trials (15.9\%) reported an FNR of 0.0 , whereas 26 trials $(37.7 \%)$ reported an FNR $>10 \%$. Significant inverse correlations were observed between the FNR and both the number of patients studied $(r=-0.42 ; P<0.01)$ and the proportion of patients who had successfully mapped SLNs nodes $(r=-0.32 ; P=0.009)$.

It is important to note, that with the acceptance of SLNB and the resulting increased histopathological scrutiny of the SLNs, the identification rate of low-volume metastases has significantly increased. ${ }^{43}$ This has resulted in a stage migration, as many of the former nodenegative cases containing occult metastases are now placed into the node-positive micrometastatic group. Because of the consequences that such a stage migration may cause and the debated prognostic significance of low-volume metastases, the pathological tumor node metastasis (pTNM) classification of malignant tumors and the American Joint Committee on Cancer (AJCC) staging definitions have split the micrometastasis category into two, one consisting of the micrometastasis category per se (tumor deposits larger than $0.2 \mathrm{~mm}$, but not larger than $2 \mathrm{~mm}$ ) and the isolated tumor cell (ITC) category (tumor deposits not larger than $0.2 \mathrm{~mm}$ ). ${ }^{44}$ The distinction between ITC and micrometastasis influences treatment decisions. Micrometastases are considered true nodal metastases, and are usually treated with completion axillary dissection and adjuvant chemotherapy. On the other hand, ITCs are not considered significant for assigning stage and are generally regarded as node-negative for both staging and treatment decisions. ${ }^{45-47}$

Results of SLNB in breast cancer are clearly operator dependent and the importance of training was confirmed by multicenter trials in which breast cancer patients underwent SLNB followed by completion with ALND. ${ }^{2}$ The revised consensus statement on guidelines from the American Society of Breast Surgeons published in December 2005 recommended that individual surgeons perform at least 20 SLNB procedures validated by an ALND (or mentored by an experienced colleague) before performing SLNB of their own. ${ }^{48}$ These guidelines are strongly supported by the American Society of Clinical Oncologists (ASCO). ${ }^{47}$ In experienced hands the FNRs for SLNB is 5\% or less. When results of the SLNB are unsatisfactory, the procedure should be abandoned in favor of standard node dissection. ${ }^{47}$

Cutaneous melanoma is the sixth most common cancer in the United States. In 2007, it was estimated that approximately 60,000 individuals developed melanoma and more than 8000 succumbed to the disease. ${ }^{49}$ The prognosis is mainly determined by thickness and ulceration of the primary tumor, as well as by the presence and extent of metastases. ${ }^{50,51}$ Early diagnosis and treatment is the key to minimizing morbidity and mortality. A local excisional biopsy with a 1-2 $\mathrm{mm}$ rim of normal appearing skin is the optimal technique for lesions 
suspected of melanoma. The definitive treatment for primary cutaneous melanoma is wide local excision with the width of the normal tissue margin depending on the thickness of the lesion. ${ }^{52}$ Metastasis to regional lymph nodes is the most important prognostic factor in early-stage melanoma. ${ }^{53}$ Before the development of SLNB, the only method to identify regional nodal metastases and stage the nodal basin was elective complete lymph node dissection (CLND). However, only $20 \%$ of patients with intermediate-thickness lesions will have metastases to regional nodes. ${ }^{54}$ The large majority of patients with melanoma would therefore undergo extensive and potentially morbid surgery without benefiting in terms of improved survival. SLNB has been proposed as a minimally invasive alternative to CLND. The AJCC has incorporated the tumor status of the sentinel node into its staging system for melanoma. ${ }^{51}$ The Multicenter Selective Lymphadenectomy Trial (MSLT-I) is the largest trial to address the role of lymphatic mapping with SLNB in determining prognosis and its impact on survival. ${ }^{54}$ Initial SLN identification rate was $95.3 \%$ overall: $99.3 \%$ for the groin, 95.3\% for the axilla, and $84.5 \%$ for the neck basins. The rate of false-negative SLNB during the trial phase, as measured by nodal recurrence in a tumor-negative dissected SLN basin, decreased with increasing case volume at each center:

$10.3 \%$ forthefirst 25 casesversus $5.2 \%$ after 25 cases. There were no operative mortalities. The low $(10.1 \%)$ complication rate after SLNB increased to $37.2 \%$ with the addition of CLND; CLND also increased the severity of complications. Thus, SLNB is a safe, low-morbidity procedure for staging the regional nodal basin in early melanoma. Even after a 30-case learning phase and 25 additional SLNB cases, the accuracy continues to increase with a center's experience. The authors concluded that SLNB should become standard care for staging the regional lymph nodes of patients with primary cutaneous melanoma.

Although, the use of ${ }^{99 \mathrm{~m}} \mathrm{Tc}$-sulfur colloid for SLNB has been previously described, this is not the only kind of LS performed today. LS is the general term for a nuclear medicine procedure which allows imaging of lymphatic flow and lymph nodes after injection of a radiotracer that is absorbed by the lymphatics. One of the major advantages of LS over direct lymphangiography is that lymphatic cannulation is not required and therefore, the procedure is comparatively simpler with minimal complication rates. ${ }^{55} \mathrm{LS}$ was pioneered more than 50 years ago by Sherman et al. who demonstrated regional lymph nodes by external scintillation following the interstitial administration of ${ }^{198} \mathrm{Au}$-colloid. ${ }^{56}$ Despite the fact that the initial results were promising, the absorbed dose at the injection site was unacceptably high ${ }^{57}$ Consequently, several ${ }^{99} \mathrm{~m}$ Tc-labeled compounds were examined including antimony sulfide colloid, stannous phytate, sulfur colloid, tin colloid, albumin, and dextran. ${ }^{58-60} 99 \mathrm{~m} \mathrm{Tc}$ is generator produced and has a $6 \mathrm{~h}$ half-life; it is therefore ideal for clinical use. ${ }^{61}$ Unlike ${ }^{198} \mathrm{Au},{ }^{99 \mathrm{~m}} \mathrm{Tc}$ does not undergo beta minus decay providing favorable dosimetry. Moreover, its $140-\mathrm{skeV}$ photons are optimal for imaging using modern gamma cameras.

Among its many applications in Oncology, LS was studied in the localization of internal mammary lymph nodes for parasternal radiation therapy, ${ }^{62}$ and in staging and treatment planning of patients with lymphoma. ${ }^{63}$ However, it is in SNLB that the technique has flourished. 
The limitations of LS and blue dye, combined with advances in imaging technology and contrast media development, have prompted a search for better lymphatic imaging across a number of modalities, including CT, US, MRI and optical imaging. These novel techniques are minimally invasive and potentially offer higher spatial resolution (enabling depiction of lymphatic channels), higher temporal resolution, cross-sectional imaging capabilities and three-dimensional image reconstruction. Nanoparticle sized contrast agents can access the lymphatic system by three different routes of administration: intravenous, intra-lymphatic (direct lymphatic injection), or interstitial. Contrast agents for direct intralymphatic injection are not being developed because of the inherent difficulties in finding and cannulating the lymphatic vessels; therefore, the newer contrast agents tend to use the other two routes. Contrast agents further increase the potential to provide functional imaging - a particular advantage for cancer imaging, where anatomical depiction alone often provides insufficient information.

\section{ADDITIONAL METHODS OF LYMPHATIC IMAGING}

\section{Direct Lymphangiography}

One of the early attempts to image the lymphatic system was on the basis of oil-based iodinated dyes that were injected directly into the lymphatics of the feet. The agent then traveled through the leg lymphatics to the abdominal lymphatics, whereupon direct radiographs or CT images could be obtained. This technique, known as 'lymphangiography' was classically used in the assessment of nodal metastases in lymphoma and a limited number of other malignancies. Its development preceded the widespread use of CT and MRI, which have all but replaced it. Lymphangiography requires great skill and expertise. Initially, a blue dye is injected intradermally into the interdigital space, in order to stain and localize the deep lymphatic vessels. An incision is then made in the skin of the foot to locate lymphatic vessels large enough to allow cannulation with a fine needle; following this an oily iodinated dye was injected into the lymphatic vessels using a mechanical pump, over a period of 60-90 min. Serial x-rays or CT scans were obtained to visualize the anatomy and, to some extent, the rate of lymphatic flow. The initial scan showed the lymphatic vessels, while scans taken at $24 \mathrm{~h}$ highlighted the lymph nodes. The injected contrast media was retained by the lymph nodes and images could be obtained for several years after injection. However, lymphangiography, in addition to being invasive, and requiring skilled practitioners, could also cause life threatening complications such as pulmonary embolization, pulmonary edema, and adult respiratory distress syndrome if the oily dye was inadvertently taken up in the veins. ${ }^{64,65}$ The invasiveness of this procedure, its expense and inconvenience for the patient, combined with a small but definite risk of severe complications, has all but eliminated lymphangiography from the modern imaging armory.

\section{Sentinel Node Imaging with CT}

Recently, multi-slice CT has been attempted for sentinel node imaging using a low molecular weight iodinated contrast agent (iopamidol). In a study performed by Suga et al., 17 patients with breast cancer underwent thin-section, three-dimensional CT after subcutaneous injection of iopamidol in the peri-tumoral and peri-areolar areas. ${ }^{66}$ Perioperative blue-dye injection was performed for comparison. CT imaging allowed 
localization of SLNs in all patients by opacifying the direct connection between the lymphatic vessel draining the injection site and an SLN. Minato et al. were also able to predict SLNs in 13/15 patients with breast cancer, either by enhancement of the lymphatic vessels draining into the SLN, or enhancement in the SLN itself, correlating well with bluedye detection. ${ }^{67}$ A problem with iopamidol-based SLN imaging is that the low molecular weight of the agent, leads to rapid opacification and early wash out from the nodes, leaving only a small temporal window for imaging. Another major disadvantage of CT imaging is that it does not provide real time guidance to the operating surgeon during identification and resection of the sentinel node.

\section{Sentinel Node Imaging with Ultrasound}

US using microbubbles as a contrast agent has been adapted for SLN imaging. Microbubbles are gas filled liposomes that are typically $5-10 \mu \mathrm{m}$ in diameter. Choi et al., injected different microbubble agents subcutaneously into rabbits. ${ }^{68}$ The agents, such as 'AF0150' were small enough in diameter to enter the lymphatic system, presumably via gaps between the lymphatic endothelial cells. All agents rapidly and markedly enhanced the popliteal lymph node following foot pad injection and hind limb massage. In addition, the lymphatic ducts were visible after interstitial injection of the microbubbles. The main disadvantages of US are its poor spatial resolution, the large size of the microbubble and hence its slower rate of uptake, its limited use in the thorax and deep retroperitoneum, and its dependence on operator experience.

\section{Optical Imaging}

Optical imaging is a rapidly advancing branch of medical imaging. It does not require ionizing radiation exposure, and can be imaged with relatively low cost and portable equipment. It is easily incorporated into the operating theatre for SLN biopsies. The near infra-red (NIR) spectrum is often used for in vivo imaging because hemoglobin, muscle and fat are least absorbent in this light range, allowing light arising from deeper tissue planes to be imaged. ${ }^{69}$ Fluorescent probes are safe and can be bound to various macromolecules, including antibodies. The main disadvantage remains the poor depth sensitivity of the technique, and penetration beyond $1-2 \mathrm{~cm}$ is currently unrealistic; however, in many cases this may be adequate for clinical use, especially in the context of identifying superficial SLNs in melanoma surgery (e.g., sentinel node imaging).

A special kind of optical fluorophore is known as a quantum dot (Qdot). Qdots are semiconductor crystals in the nanometer size ranging between 5 and $20 \mathrm{~nm}$ in diameter. They have high quantum yield and are therefore very bright. The wavelength of emitted energy of Qdots can be controlled by changing their size and shape; therefore, they can emit light in the NIR spectrum. ${ }^{70}$ In addition to their high light output, Qdots can be excited with broadband light below the emission wavelength. Moreover, because of their narrow emission bandwidth, multiple Qdots can be used simultaneously with the same excitation light. In comparison, organic fluorophores usually require specific excitation light near to the emission wavelength limiting the number of dyes that can be simultaneously excited and detected. ${ }^{71}$ On the other hand, the main disadvantage of Qdots is their potential toxicity due to their cadmium-selenium or cadmium-tellurium core. ${ }^{72}$ Additionally, Qdots can induce 
cell death by formation of reactive oxygen species as in some cases Qdots can transfer absorbed optical energy to oxygen radicals, which may cause DNA damage, potentially resulting in apoptosis and necrosis. ${ }^{73}$ However, their clinical feasibility is yet to be determined as the overall dose is extremely low and Qdots can be designed to avoid creation of reactive oxygen species.

The range of maximum fluorescence of Qdots is determined by their elemental composition extending over the whole visible and NIR spectrum. ${ }^{73}$ The size of Qdots makes them ideal agents for lymphatic imaging via interstitial injection. In order to make Qdots suitable for lymphatic imaging, their surfaces have to be modified. ${ }^{73}$

Qdots are synthesized in nonpolar organic solvents and are solubilized by hydrophobic surface ligands such as aliphatic phosponines, fatty acids, phosphonine oxides, and aliphatic amines. ${ }^{73}$ Modification of the surface with a hydrophilic coating facilitates solubilization of Qdots in aqueous media and their aggregation. ${ }^{73}$ Exchanging hydrophobic ligands with thiol molecules, dendrons, peptides, or phosphines, encapsulating Qdots in a silica shell and using interactions between Qdots and amphiphilic polymers, phospholipids are some of the strategies for modifying them for in vivo use. ${ }^{73} \mathrm{~A}$ furtherstep is conjugation with biomolecules like peptides or proteins that can target cell surface receptors. ${ }^{73}$

Kim et al. reported successful in vivo imaging of the lymphatics using NIR Qdots in order to detect SLNs arising from breast tissue. ${ }^{74}$ Retention of Qdots in lymph nodes was shown by several studies in animal models. ${ }^{75-77}$ Parungo et al. reported use of Qdots in the detection of lymphatic drainage of peritoneal and pleural spaces in animal models. ${ }^{78,79} \mathrm{Knapp}$ et al. used NIR Qdots to demonstrate SLNs in invasive urinary bladder cancer in animal models. ${ }^{80}$ Additionally, utility of Qdots in SLN mapping of gastrointestinal tract and lung was reported by Soltesz et al. ${ }^{81,82}$

Hama et al. have shown utility of fluorescence lymphangiography using two NIR Qdots with different emission spectra. ${ }^{83}$ Recently, Kobayashi et al. demonstrated simultaneous multicolor fluorescence imaging of five different lymphatic basins using five NIR Qdots (Figure 4). ${ }^{84}$ This is only possible with Qdots because of their narrow emission wavelengths and broad excitation tolerance. Fluorescence imaging enables improved sensitivity for lymph nodes close enough to surface to be imaged specifically during surgery; moreover the combination of Qdot fluorescence imaging with other imaging modalities such as MRI, CT, scintigraphy and positron emission tomography may allow mapping of deeper lymph nodes. 74

\section{Magnetic Resonance Lymphography}

The use of macromolecular agents employing gadolinium labeling is at a relatively early stage of research. Some groups have demonstrated the feasibility of magnetic resonance lymphography (MRL) with Gd containing liposomes in animal models. ${ }^{85,86}$ Good uptake was demonstrated in regional lymph nodes following subcutaneous injection, which is likely related to trapping of the liposomes by macrophages. Misselwitz et al. used the macromolecular contrast medium Gadomer-17 to image the inguinal and iliac nodes in dogs following hind limb injection. ${ }^{87}$ Enhancement was seen 15 min post-injection, but was 
maximal 60-90 min after injection, with signal enhancement increasing by as much as 450 $960 \%$, depending on the initial dose.

Multiple studies have evaluated the use of ultrasmall superparamagnetic particles of iron oxide (USPIO) for detection of nodal metastases. ${ }^{88}$ When injected intravenously, USPIO particles are transported into the interstitial space reaching normal functioning lymph nodes where they are ultimately phagocitozed by macrophages. The magnetic properties of USPIO are such that it generates susceptibility artifacts (signal loss) and normal lymph nodes appear dark (i.e., negative contrast). In lymph nodes that contain metastases, tumor displaces macrophages, preventing uptake of USPIO. In a novel approach in the detection of SLNs, Torchia and colleagues performed interstitial and intradermal injections of USPIO (ferumoxtran-10) in anesthetized pigs. ${ }^{89}$ Ferumoxtran-10 (Combidex ${ }^{\circledR}$, Advanced Magnetics Incorporated, Cambridge, MA) is a first generation USPIO nanoparticle consistent of an iron-oxide crystalline core coated with a layer of dextran. The particle size is approximately $20 \mathrm{~nm}$. After injection of $0.2-5 \mathrm{mg}$ of ferumoxtran-10, sequential MRI images were obtained starting at $15 \mathrm{~min}$ post injection to as late as $48 \mathrm{~h}$. SLNs were classified as the first lymph node(s) to show susceptibility artifacts. In this study, detection of SLNs by MRI imaging compared favorably with intraoperative isosulfan blue.

It is also possible to use MRI to image the lymphatic-convective transport in vivo. Pathak et al. selected two murine breast cell lines, known to have differences in invasiveness. ${ }^{90}$ Using albumin-Gd-DTA as a contrast agent they were able to classify ROIs as 'pooling' if the macromolecular contrast media (MMCM) concentration increased over time,or 'draining' if it decreased relative to early phase images. The more invasive tumor line had a significantly higher MRI-detected number of 'draining' voxels. Thus, the lymphatic drainage pattern correlates with the metastasis rate and lymphangiogenesis. Drainage may be dependent on both the 'invasiveness' of the tumor and on the extracellular matrix integrity which, if reduced, can facilitate passage of tumor cells along with extracellular fluid.

\section{Dendrimers for Sentinel Lymph Node Imaging}

Dendrimers are monodispersed synthetically produced organic polymers. They can be produced at precise but different chemically identical sizes. Two forms of dendrimers are commercially available: polyamidoamine (PAMAM) and diaminobutane (DAB) core polypropylimine (PPI). Different generations (sizes) of dendrimers have been investigated as MRL macromolecular contrast agents. Kobayashi et al. used interstitially injected generation-6 (G6) PAMAM dendrimers loaded with gadolinium chelates (Gd) to image the lymphatic system and the sentinel nodes of normal mice and mice with xenografted breast tumors. ${ }^{91}$ Gd-G6 dendrimer was injected directly into the mammary gland or peri-tumorally, imaged by 3D T1 weighted MRI and 3D reconstruction was used to aid anatomical localization. The same group used a similar contrast agent in a mouse lymphoma model. ${ }^{92}$ They were able to differentiate normal and abnormal lymphatics and distinguish intralymphatic from extralymphatic disease. Kobayashi et al. also compared MRL with either Gd-PAMAM dendrimers of different generations, or the less hydrophilic Gd-DAB generations in murine models. ${ }^{93}$ Gd-PAMAM-G8was retained in the fine lymphatic vessels without major leakage, resulting in excellent imaging of the lymphatic channels (Figure 5). 
However, Gd-PAMAM-G4 provided better localization of lymph nodes that were close to the liver, because of a reduced background signal. DAB dendrimers are expected to be more rapidly cleared from the circulation because of their uptake and excretion by the liver and kidneys. Thus, DAB-G5 was cleared more rapidly from the lymphatic vessels, but retained in the lymph nodes. Therefore, PAMAM dendrimers may be better for imaging the lymphatics and $\mathrm{DAB}$ dendrimers more optimal for lymph node imaging.

Another interesting advance in lymph node imaging is the use of dual-modality contrast agents. Talanov et al. synthesized a PAMAM-G6 dendrimer conjugated to Gadolinium for MRL and Cy5.5, a NIR dye for optical imaging. ${ }^{94}$ The agent was injected into the mammary fat pad of mice and SLNs were successfully imaged on MRI, followed by optical imaging (Figure 6). MR has a number of advantages, including good spatial resolution and lack of ionizing radiation exposure while optical imaging offers portability to the operating theatre. Despite the promise of MMCMs for use in MRL, USPIOs are the only agents that have been tried in humans. Iron oxide based contrast agents seem ideally suited to lymph node imaging when used for staging purposes and progress is certain to be made in this field. However, for purposes of identifying the sentinel node, interstitial injection of gadolinium based macromolecules, such as dendrimers seem to be a superior choice. The further advances in contrast agent development may eventually lead to substantial progress in this field. One concern that places the future in doubt is the possibility of gadolinium chelates leaching from interstitially injected macromolecules and dissociating in the interstitial tissues. A new syndrome, found mainly in patients with renal failure who have received intravenous injections of gadolinium chelates, produces severe interstitial fibrosis and is known as nephrogenic sclerosing fibrosis (NSF).${ }^{95,96}$ Its origins have been traced to free gadolinium in the interstitial tissues because of the slow clearance of the chelate in patients with renal failure. To be sure, the exact pathogenesis of NSF remains obscure; however, its recognition has had a chilling effect on the development of new gadolinium containing compounds, particularly those that are to be injected interstitially. Certainly, there is the theoretical risk of gadolinium leaching from interstitially injected macromolecules with the potential for unknown toxic events. For this reason, the field is currently progressing cautiously and potential side effects are being evaluated in animal trials.

\section{CONCLUSIONS}

Currently, sentinel node imaging and biopsy is a vital part of the workup of some cancers in humans. Current methods have limitations including lack of depth and skin staining (blue dye), poor spatial resolution, and exposure to ionizing radiation (LS). It is conceivable that in the near future nanoparticles for optical imaging or MRI will offer the ability to perform SLN imaging in vivo without these disadvantages. The ideal agent for identifying SLNs would be delivered in high concentration to the lymph nodes to enable imaging and would have a low background concentration in the surrounding normal tissues. Additionally, the ideal agent would concentrate in the SLN quickly after injection depicting the lymphatic drainage and remaining in place for intraoperative localization. Such an agent could also be considered a surrogate marker for a similar nanoparticle containing a cytotoxic payload, e.g., anticancer drugs and a tumor vaccine to induce an immune response or to act as a target for 
neutron capture therapy (NCT). Thus, SLN imaging may one day be incorporated into the delivery of regional therapy.

\section{REFERENCES}

1. Moghimi SM, Hunter AC, Murray JC. Nanomedicine: current status and future prospects. Faseb J 2005, 19(3):311-330. [PubMed: 15746175]

2. Krag D, Weaver D, Ashikaga T, Moffat F, Klimberg VS, et al. The sentinel node in breast cancer-a multicenter validation study. N Engl J Med 1998, 339(14):941-946. [PubMed: 9753708]

3. Early Breast Cancer Trialists' Collaborative Group(EBCTCG). Effects of chemotherapy and hormonal therapy for early breast cancer on recurrence and 15-year survival: an overview of the randomised trials. Lancet 2005, 365(9472):1687-1717. [PubMed: 15894097]

4. Morton DL, Wen DR, Wong JH, Economou JS, Cagle LA, et al. Technical details of intraoperative lymphatic mapping for early stage melanoma. Arch Surg 1992, 127(4):392-399. [PubMed: 1558490]

5. Cabanas RM. An approach for the treatment of penile carcinoma. Cancer 1977, 39(2):456-466. [PubMed: 837331]

6. Beaulac SM, McNair LA, Scott TE, LaMorte WW, Kavanah MT. Lymphedema and quality of life in survivors of early-stage breast cancer. Arch Surg 2002, 137(11):1253-1257. [PubMed: 12413312]

7. Luciani A, Itti E, Rahmouni A, Meignan M, Clement O. Lymph node imaging: basic principles. Eur J Radiol 2006, 58(3):338-344. [PubMed: 16473489]

8. Cserni G Metastases in axillary sentinel lymph nodes in breast cancer as detected by intensive histopathological work up. J Clin Pathol 1999, 52(12):922-924. [PubMed: 10711258]

9. Rodier JF, Velten M, Wilt M, Martel P, Ferron G, et al. Prospective multicentric randomized study comparing periareolar and peritumoral injection of radiotracer and blue dye for the detection of sentinel lymph node in breast sparing procedures: FRANSENODE trial. J Clin Oncol 2007, 25(24): 3664-3669. [PubMed: 17485709]

10. Eshima D, Eshima LA, Gotti NM, Herda SC, Algozine CA, et al. Technetium-99 m-sulfur colloid for lymphoscintigraphy: effects of preparation parameters. J Nucl Med 1996, 37(9):1575-1578. [PubMed: 8790222]

11. Goldfarb LR, Alazraki NP, Eshima D, Eshima LA, Herda SC, et al. Lymphoscintigraphic identification of sentinel lymph nodes: clinical evaluation of 0.22 -micron filtration of Tc-99 m sulfur colloid. Radiology 1998, 208(2):505-509. [PubMed: 9680583]

12. Sakorafas GH, Peros G. Sentinel lymph node biopsy in breast cancer: what a physician should know, a decade after its introduction in clinical practice. Eur J Cancer Care (Engl) 2007, 16(4): 318-321. [PubMed: 17587354]

13. Montgomery LL, Thorne AC, Van Zee KJ, Fey J, Heerdt AS, et al. Isosulfan blue dye reactions during sentinel lymph node mapping for breast cancer. Anesth Analg 2002, 95(2):385-388, table of contents. [PubMed: 12145056]

14. Somasundaram SK, Chicken DW, Keshtgar MR. Detection of the sentinel lymph node in breast cancer. Br Med Bull 2007, 84:117-131. [PubMed: 18174216]

15. Paleri V, Rees G, Arullendran P, Shoaib T, Krishman S. Sentinel node biopsy in squamous cell cancer of the oral cavity and oral pharynx: a diagnostic meta-analysis. Head Neck 2005, 27(9): 739-747. [PubMed: 16047368]

16. Gupta SG, Wang LC, Penas PF, Gellenthin M, Lee SJ, et al. Sentinel lymph node biopsy for evaluation and treatment of patients with Merkel cell carcinoma: The Dana-Farber experience and meta-analysis of the literature. Arch Dermatol 2006, 142(6):685-690. [PubMed: 16785370]

17. Perdona S, Autorino R, Gallo L, Di Lorenzo G, Cascini GL, et al. Role of dynamic sentinel node biopsy in penile cancer: our experience. J Surg Oncol 2006, 93(3):181-185. [PubMed: 16482606]

18. Scheidler J, Hricak H, Vigneron DB, Yu KK, Sokolov DL, et al. Prostate cancer: Localization with three-dimensional proton MR spectroscopic imaging-Clinicopathologic study. Radiology 1999, 213(2):473-480. [PubMed: 10551229] 
19. Jamieston J, Dobson J. Lectures on the Lymphatic system of the stomach. Lancet 1907, 1:10611062.

20. Alex JC, Krag DN. Gamma-probe guided localization of lymph nodes. Surg Oncol 1993, 2(3):137143. [PubMed: 8252203]

21. Krag DN, Weaver DL, Alex JC, Fairbank JT. Surgical resection and radiolocalization of the sentinel lymph node in breast cancer using a gamma probe. Surg Oncol 1993, 2(6):335-339, discussion 340. [PubMed: 8130940]

22. Giuliano AE, Kirgan DM, Guenther JM, Morton DL. Lymphatic mapping and sentinel lymphadenectomy for breast cancer. Ann Surg 1994, 220(3):391-398, discussion 398-401. [PubMed: 8092905]

23. Albertini JJ, Lyman GH, Cox C, Yeatman T, Balducci L, et al. Lymphatic mapping and sentinel node biopsy in the patient with breast cancer. Jama 1996, 276(22):1818-1822. [PubMed: 8946902]

24. Larson SM, Nelp WB. Radiopharmacology of a simplifield technetium-99 m-colloid preparation for photoscanning. J Nucl Med 1966, 7(11):817-826. [PubMed: 5955361]

25. Bergqvist L, Strand SE, Persson BR. Particle sizing and biokinetics of interstitial lymphoscintigraphic agents. Semin Nucl Med 1983, 13(1):9-19. [PubMed: 6220471]

26. Tsopelas C Understanding the radiolabelling mechanism of $99 \mathrm{mTc}$-antimony sulphide colloid. Appl Radiat Isot 2003, 59(5-6):321-328. [PubMed: 14622929]

27. Tassenoy A, van der Veen P, Bossuyt A, Lamote J, Lievens P. Lymphatic pathways of the upper medial quadrant of the breast in healthy women: radiotracer study of the sentinel lymph node. Lymphology 2002, 35(4):153-160. [PubMed: 12570324]

28. Alazraki N, Glass EC, Castronovo F, Olmos RA, Podoloff D. Procedure guideline for lymphoscintigraphy and the use of intraoperative gamma probe for sentinel lymph node localization in melanoma of intermediate thickness 1.0. J Nucl Med 2002, 43(10):1414-1418. [PubMed: 12368382]

29. Maza S, Valencia R, Geworski L, Zander A, Guski H, et al. Peritumoural versus subareolar administration of technetium-99 m nanocolloid for sentinel lymph node detection in breast cancer: preliminary results of a prospective intra-individual comparative study. Eur J Nucl Med Mol Imaging 2003, 30(5):651-656. [PubMed: 12612811]

30. Maza S, Thomas A, Winzer KJ, Huttner C, Blohmer JU, et al. Subareolar injection of technetium-99 m nanocolloid yields reliable data on the axillary lymph node tumour status in breast cancer patients with previous manipulations on the primary tumour: a prospective study of 117 patients. Eur J Nucl Med Mol Imaging 2004, 31(5):671-675. [PubMed: 14745517]

31. Yeung HW, Cody IH, Turlakow A, Riedel ER, Fey J, et al. Lymphoscintigraphy and sentinel node localization in breast cancer patients: a comparison between 1-day and 2-day protocols. J Nucl Med 2001, 42(3):420-423. [PubMed: 11337517]

32. Mar MV, Miller SA, Kim EE, Macapinlac HA. Evaluation and localization of lymphatic drainage and sentinel lymph nodes in patients with head and neck melanomas by hybrid SPECT/CT lymphoscintigraphic imaging. J Nucl Med Technol 2007, 35(1):10-16quiz 17-20.

33. Smith RA, Duffy SW, Gabe R, Tabar L, Yen AM, Chen TH. The randomized trials of breast cancer screening: what have we learned? Radiol Clin North Am 2004, 42(5):793-806, v. [PubMed: 15337416]

34. Bijker N, Meijnen P, Peterse JL, Bogaerts J, Van Hoorebeeck I, et al. Breast-conserving treatment with or without radiotherapy in ductal carcinoma-in-situ: ten-year results of European Organisation for Research and Treatment of Cancer randomized phase III trial 10853 - a study by the EORTC Breast Cancer Cooperative Group and EORTC Radiotherapy Group. J Clin Oncol 2006, 24(21):3381-3387. [PubMed: 16801628]

35. Clarke M, Collins R, Darby S, Davies C, Elphinstone P, et al. Effects of radiotherapy and of differences in the extent of surgery for early breast cancer on local recurrence and 15-year survival: an overview of the randomised trials. Lancet 2005, 366(9503):2087-2106. [PubMed: 16360786]

36. Nemoto T, Vana J, Bedwani RN, Baker HW, McGregor FH, et al. Management and survival of female breast cancer: results of a national survey by the American College of Surgeons. Cancer 1980, 45(12):2917-2924. [PubMed: 7388735] 
37. Carter CL, Allen C, Henson DE. Relation of tumor size, lymph node status, and survival in 24,740 breast cancer cases. Cancer 1989, 63(1):181-187. [PubMed: 2910416]

38. Mustafa IA, Cole B, Wanebo HJ, Bland KI, Chang HR. Prognostic analysis of survival in small breast cancers. J Am Coll Surg 1998, 186(5):562-569. [PubMed: 9583697]

39. Rivadeneira DE, Simmons RM, Christos PJ, Hanna K, Daly JM, et al. Predictive factors associated with axillary lymph node metastases in T1a and T1b breast carcinomas: analysis in more than 900 patients. J Am Coll Surg 2000, 191(1):1-6, discussion 6-8. [PubMed: 10898177]

40. Mariani G, Moresco L, Viale G, Villa G, Bagnasco M, et al. Radioguided sentinel lymph node biopsy in breast cancer surgery. J Nucl Med 2001, 42(8):1198-1215. [PubMed: 11483681]

41. Martin C, Cutuli B, Velten M. Predictive model of axillary lymph node involvement in women with small invasive breast carcinoma: axillary metastases in breast carcinoma. Cancer 2002, 94(2):314322. [PubMed: 11900217]

42. Kim T, Giuliano AE, Lyman GH. Lymphatic mapping and sentinel lymph node biopsy in earlystage breast carcinoma: a metaanalysis. Cancer 2006, 106(1):4-16. [PubMed: 16329134]

43. Chen SL, Hoehne FM, Giuliano AE. The prognostic significance of micrometastases in breast cancer: a SEER population-based analysis. Ann Surg Oncol 2007, 14(12):3378-3384. [PubMed: 17899293]

44. Sobin LH, Hermanek P, Hutter RV. TNM classification of malignant tumors. A comparison between the new (1987) and the old editions. Cancer 1988, 61(11):2310-2314. [PubMed: 3284634]

45. Mittendorf EA, Hunt KK. Significance and management of micrometastases in patients with breast cancer. Expert Rev Anticancer Ther 2007, 7(10):1451-1461. [PubMed: 17944569]

46. Schwartz GF, Giuliano AE, Veronesi U. Proceedings of the consensus conference on the role of sentinel lymph node biopsy in carcinoma of the breast, April 19-22, 2001, Philadelphia, Pennsylvania. Cancer 2002, 94(10):2542-2551. [PubMed: 12173319]

47. Lyman GH, Giuliano AE, Somerfield MR, Benson AB, III, Bodurka DC, et al. American Society of Clinical Oncology guideline recommendations for sentinel lymph node biopsy in early-stage breast cancer. J Clin Oncol 2005, 23(30):7703-7720. [PubMed: 16157938]

48. Americal Society of Breast Surgeons. Guidelines from the Americal Society of Breast Surgeons, avaliable on line at http://www.breastsurgeons.org/slnd.shtml (accessed September 15, 2008).

49. Jemal A, Siegel R, Ward E, Murray T, Xu J, et al. Cancer Statistics, 2007 CA Cancer J Clin 2007, 57(1):43-66. [PubMed: 17237035]

50. Gimotty PA, Botbyl J, Soong SJ, Guerry D. A population-based validation of the American Joint Committee on Cancer melanoma staging system. J Clin Oncol 2005, 23(31):8065-8075. [PubMed: 16258105]

51. Balch CM, Buzaid AC, Soong SJ, Atkins MB, Cascinelli N, et al. Final version of the American Joint Committee on Cancer staging system for cutaneous melanoma. J Clin Oncol 2001, 19(16): 3635-3648. [PubMed: 11504745]

52. Cascinelli N Margin of resection in the management of primary melanoma. Semin Surg Oncol 1998, 14(4):272-275. [PubMed: 9588719]

53. Balch CM, Soong SJ, Gershenwald JE, Thompson JF, Reintgen DS, et al. Prognostic factors analysis of 17,600 melanoma patients: validation of the American Joint Committee on Cancer melanoma staging system. J Clin Oncol 2001, 19(16):3622-3634. [PubMed: 11504744]

54. Morton DL, Cochran AJ, Thompson JF, Elashoff R, Essner R, et al. Sentinel node biopsy for earlystage melanoma: accuracy and morbidity in MSLT-I, an international multicenter trial. Ann Surg 2005, 242(3):302-311, discussion 311-3. [PubMed: 16135917]

55. Winterer JT, Blum U, Boos S, Konstantinides S, Langer M. Cerebral and renal embolization after lymphography in a patient with non-Hodgkin lymphoma: case report. Radiology 1999, 210(2): 381-383. [PubMed: 10207418]

56. Sherman AI, Ter-Pogossian M. Lymph-node concentration of radioactive colloidal gold following interstitial injection. Cancer 1953, 6(6):1238-1240. [PubMed: 13106841]

57. Zum WK. Lymphologie mit Radionukliden. Verlag Hildergard Hoffman 1972, 30-32(Berlin).

58. Juma N, Andrey T, Ege GN. Comparison as a lymphoscintigraphic agent between 99Tcm dextran and 99Tcm antimony sulphide colloid. Br J Radiol 1985, 58(688):325-330. [PubMed: 2415201] 
59. Ege GN, Warbick A. Lymphoscintigraphy: a comparison of 99Tc(m) antimony sulphide colloid and 99Tc(m) stannous phytate. Br J Radiol 1979, 52(614):124-129. [PubMed: 427362]

60. Strand SE, Persson BR. Quantitative lymphoscintigraphy I: Basic concepts for optimal uptake of radiocolloids in the parasternal lymph nodes of rabbits. J Nucl Med 1979, 20(10):1038-1046. [PubMed: 231640]

61. Allen JF. An Improved Technetium-99 m Generator for Medical Applications. Int J Appl Radiat Isot 1965, 16:332-334. [PubMed: 14313712]

62. Dufresne EN, Kaplan WD, Zimmerman RE, Rose CM. The application of internal mammary lymphoscintigraphy to planning of radiation therapy. J Nucl Med 1980, 21(7):697-699. [PubMed: 7391847]

63. Pearlman AW. Abdominal lymph node scintiscanning with radioactive gold (Au198) for evaluation and treatment of patients with lymphoma. Am J Roentgenol Radium Ther Nucl Med 1970, 109(4): 780-792.

64. Derveaux L, Van Mieghem W, Billiet L. Severe pulmonary thromboembolism following lymphography. Acta Clin Belg 1983, 38(2):119-121. [PubMed: 6306969]

65. Silvestri RC, Huseby JS, Rughani I, Thorning D, Culver BH. Respiratory distress syndrome from lymphangiography contrast medium. Am Rev Respir Dis 1980, 122(4):543-549. [PubMed: 6254413]

66. Suga K, Yuan Y, Okada M, Matsunaga N, Tangoku A et al. Breast sentinel lymph node mapping at CT lymphography with iopamidol: preliminary experience. Radiology 2004, 230(2):543-552. [PubMed: 14699178]

67. Minato M, Hirose C, Sasa M, Nishitani H, Hirose Y, et al. 3-dimensional computed tomography lymphography-guided identification of sentinel lymph nodes in breast cancer patients using subcutaneous injection of nonionic contrast medium: a clinical trial. J Comput Assist Tomogr 2004, 28(1):46-51. [PubMed: 14716231]

68. Choi SH, Kono Y, Corbeil J, Lucidarme O, Mattrey RF. Model to quantify lymph node enhancement on indirect sonographic lymphography. AJR Am J Roentgenol 2004, 183(2):513517. [PubMed: 15269049]

69. Carrington C Optical imaging sheds light on cancer's signature-regional blood flow and tissue oxygenization measures may permit breast cancer detection. Diagn Imaging 2004, (accessed June), www.diagnosticimaging.com/news/display/article/113619/1185817.

70. Barrett T, Choyke PL, Kobayashi H. Imaging of the lymphatic system: new horizons. Contrast Media Mol Imaging 2006, 1(6):230-245. [PubMed: 17191764]

71. Dahan M, Laurence T, Pinaud F, Chemla DS, Alivisatos AP, et al. Time-gated biological imaging by use of colloidal quantum dots. Opt Lett 2001, 26(11):825-827. [PubMed: 18040463]

72. Derfus A, Chan W, Bhatia S. Probing the cytotoxicity of semiconductor quantum dots. Nano Lett 2004, 4:11-18. [PubMed: 28890669]

73. Hild WA, Breunig M, Goepferich A. Quantum dots-nano-sized probes for the exploration of cellular and intracellular targeting. Eur J Pharm Biopharm 2008, 68(2):153-168. [PubMed: 17869074]

74. Kim S, Lim YT, Soltesz EG, De Grand AM, Lee J, et al. Near-infrared fluorescent type II quantum dots for sentinel lymph node mapping. Nat Biotechnol 2004, 22(1):93-97. [PubMed: 14661026]

75. Gopee NV, Roberts DW, Webb P, Cozart CR, Siitonen PH, et al. Migration of intradermally injected quantum dots to sentinel organs in mice. Toxicol Sci 2007, 98(1):249-257. [PubMed: 17404394]

76. Ballou B, Ernst LA, Andreko S, Harper T, Fitzpatrick JA, et al. Sentinel lymph node imaging using quantum dots in mouse tumor models. Bioconjug Chem 2007, 18(2):389-396. [PubMed: 17263568]

77. Frangioni JV, Kim SW, Ohnishi S, Kim S, Bawendi MG. Sentinel lymph node mapping with typeII quantum dots. Methods Mol Biol 2007, 374:147-159. [PubMed: 17237537]

78. Parungo CP, Soybel DI, Colson YL, Kim SW, Ohnishi S, et al. Lymphatic drainage of the peritoneal space: a pattern dependent on bowel lymphatics. Ann Surg Oncol 2007, 14(2):286-298. [PubMed: 17096058] 
79. Parungo CP, Colson YL, Kim SW, Kim S, Cohn LH, et al. Sentinel lymph node mapping of the pleural space. Chest 2005, 127(5):1799-1804. [PubMed: 15888861]

80. Knapp DW, Adams LG, Degrand AM, Niles JD, Ramos-Vara JA, et al. Sentinel lymph node mapping of invasive urinary bladder cancer in animal models using invisible light. Eur Urol 2007, 52(6):1700-1708. [PubMed: 17646044]

81. Soltesz EG, Kim S, Kim SW, Laurence RG, De Grand AM, et al. Sentinel lymph node mapping of the gastrointestinal tract by using invisible light. Ann Surg Oncol 2006, 13(3):386-396. [PubMed: 16485157]

82. Soltesz EG, Kim S, Laurence RG, DeGrand AM, Parungo CP, et al. Intraoperative sentinel lymph node mapping of the lung using near-infrared fluorescent quantum dots. Ann Thorac Surg 2005, 79(1):269-277, discussion 269-77. [PubMed: 15620956]

83. Hama Y, Koyama Y, Urano Y, Choyke PL, Kobayashi H. Two-color lymphatic mapping using Igconjugated near infrared optical probes. J Invest Dermatol 2007, 127(10):2351-2356. [PubMed: 17522707]

84. Kobayashi H, Hama Y, Koyama Y, Barrett T, Regino CA, et al. Simultaneous multicolor imaging of five different lymphatic basins using quantum dots. Nano Lett 2007, 7(6):1711-1716. [PubMed: 17530812]

85. Fujimoto Y, Okuhata Y, Tyngi S, Namba Y, Oku N. Magnetic resonance lymphography of profundus lymph nodes with liposomal gadolinium-diethylenetriamine pentaacetic acid. Biol Pharm Bull 2000, 23(1):97-100. [PubMed: 10706419]

86. Misselwitz B, Sachse A. Interstitial MR lymphography using Gd-carrying liposomes. Acta Radiol Suppl 1997, 412:51-55. [PubMed: 9240081]

87. Misselwitz B, Schmitt-Willich H, Michaelis M, Oellinger JJ. Interstitial magnetic resonance lymphography using a polymeric $\mathrm{t} 1$ contrast agent: initial experience with Gadomer-17. Invest Radiol 2002, 37(3):146-151. [PubMed: 11882794]

88. Hoffman HT,Quets J,Toshiaki T, Funk GF, McCulloch TM: et al. Functional magnetic resonance imaging using iron oxide particles in characterizing head and neck adenopathy. Laryngoscope 2000, 110(9):1425-1430. [PubMed: 10983936]

89. Torchia MG, Nason R, Danzinger R, Lewis JM, Thliveris JA. Interstitial MR lymphangiography for the detection of sentinel lymph nodes. J Surg Oncol 2001, 78(3):151-156, discussion 157. [PubMed: 11745796]

90. Pathak AP, Artemov D, Neeman M, Bhujwalla ZM. Lymph node metastasis in breast cancer xenografts is associated with increased regions of extravascular drain, lymphatic vessel area, and invasive phenotype. Cancer Res 2006, 66(10):5151-5158. [PubMed: 16707438]

91. Kobayashi H, Kawamoto S, Sakai Y, Choyke PL, Star RA et al. Lymphatic drainage imaging of breast cancer in mice by micro-magnetic resonance lymphangiography using a nano-size paramagnetic contrast agent. J Natl Cancer Inst 2004, 96(9):703-708. [PubMed: 15126607]

92. Kobayashi H, Kawamoto S, Brechbiel MW, Bernardo M, Sato N: et al. Detection of lymph node involvement in hematologic malignancies using micromagnetic resonance lymphangiography with a gadolinium-labeled dendrimer nanoparticle. Neoplasia 2005, 7(11):984-991. [PubMed: 16331884]

93. Kobayashi H, Kawamoto S, Choyke PL, Sato N, Knopp MV: et al. Comparison of dendrimer-based macromolecular contrast agents for dynamic micro-magnetic resonance lymphangiography. Magn Reson Med 2003, 50(4):758-766. [PubMed: 14523962]

94. Talanov VS, Regino CA, Kobayashi H, Bernardo M, Choyke PL: et al. Dendrimer-based nanoprobe for dual modality magnetic resonance and fluorescence imaging. Nano Lett 2006, 6(7): 1459-1463. [PubMed: 16834429]

95. Grobner T Gadolinium-a specific trigger for the development of nephrogenic fibrosing dermopathy and nephrogenic systemic fibrosis?. Nephrol Dial Transplant 2006, 21(4):1104-1108. [PubMed: 16431890]

96. Marckmann P, Skov L, Rossen K, Dupont A, Damholt MB: et al. Nephrogenic systemic fibrosis: suspected causative role of gadodiamide used for contrast-enhanced magnetic resonance imaging. J Am Soc Nephrol 2006, 17(9):2359-2362. [PubMed: 16885403] 


\section{ANTERIOR}

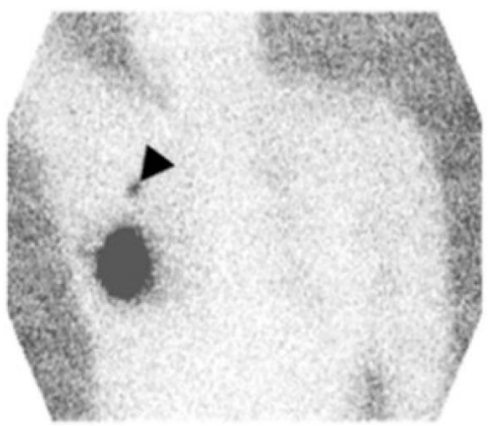

\section{LATERAL}

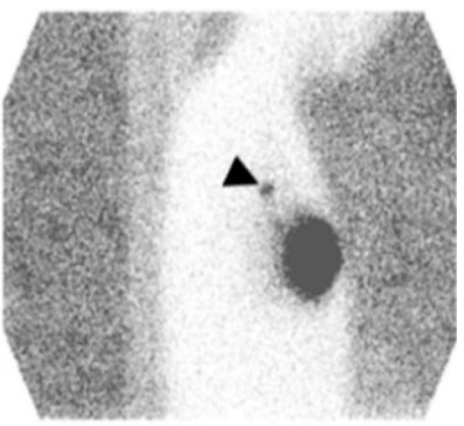

FIGURE 1 .

Anterior and lateral planar images of the chest obtained after the intradermal injection of radiotracer in the skin overlying the primary tumor. The large area of intense radiotracer uptake in the right breast corresponds to the injection site. The sentinel lymph node is clearly noted in the right axilla (arrowheads). 


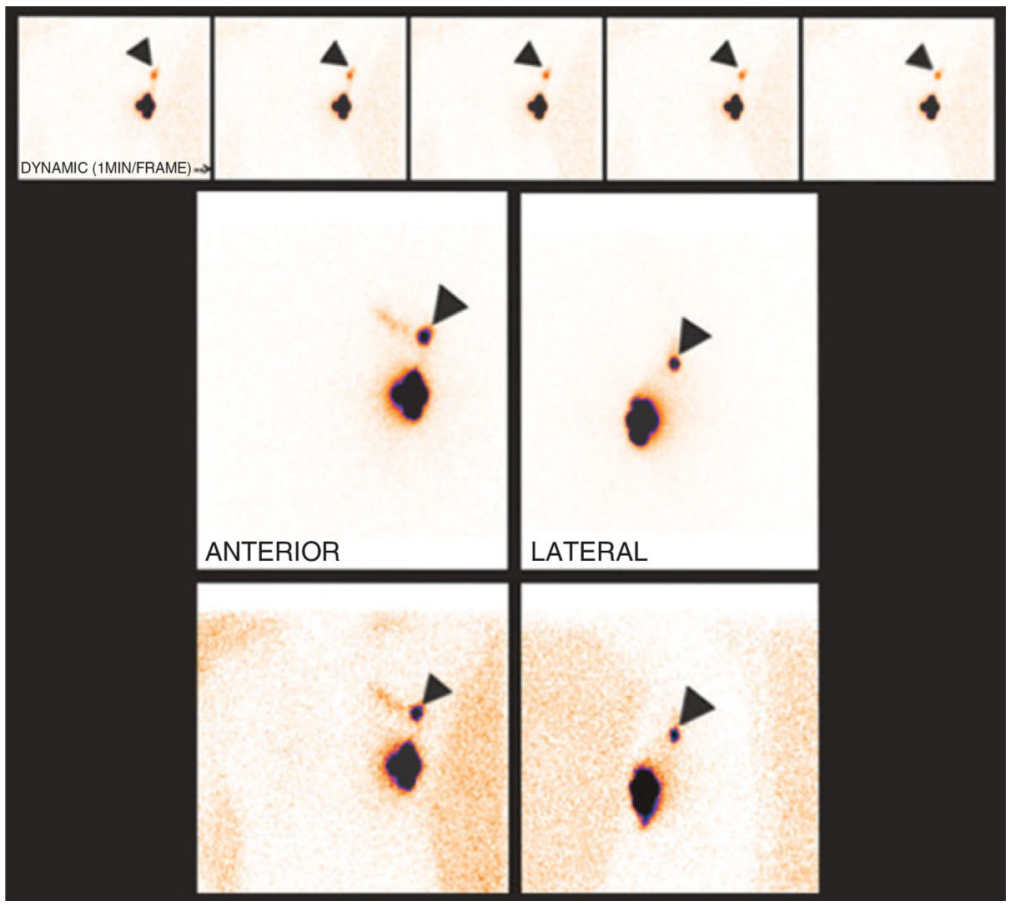

FIGURE 2 |

Dynamic (top row) and static images of the chest obtained after the subdermal injection of radiotracer around the peri-areolar area. Radiotracer is noted draining to a sentinel lymph node in the left axilla (arrowheads). Echelon lymph nodes or a lymphatic channel starts to appear medial to the sentinel node. 


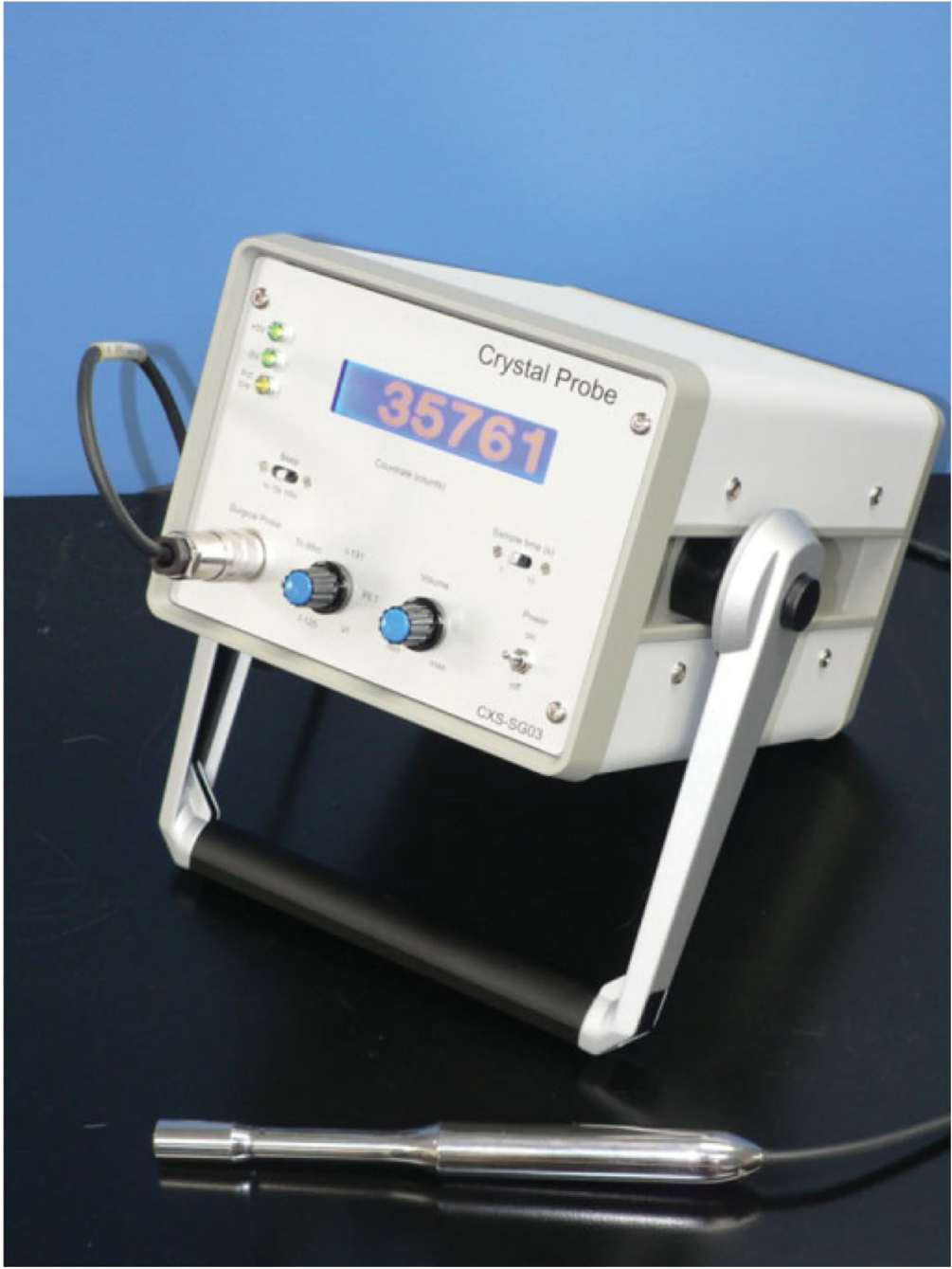

FIGURE 3 |.

Modern gamma probe system currently in use for sentinel lymph node detection (Nuclear Fields Corp., Des Plaines, Illinois). 
FIGURE 4 |.

In vivo visualization spectral fluorescence imaging of lymphatic drainage of a mouse with five carboxyl Qdots (565, blue; 605, green; 655, yellow; 705, magenta; 800, red) injected intracutaneously into the middle digits of the bilateral upper extremities, the bilateral ears, and at the median chin. Five primary draining lymph nodes are simultaneously demonstrated with different colors through the skin in the in vivo image. 


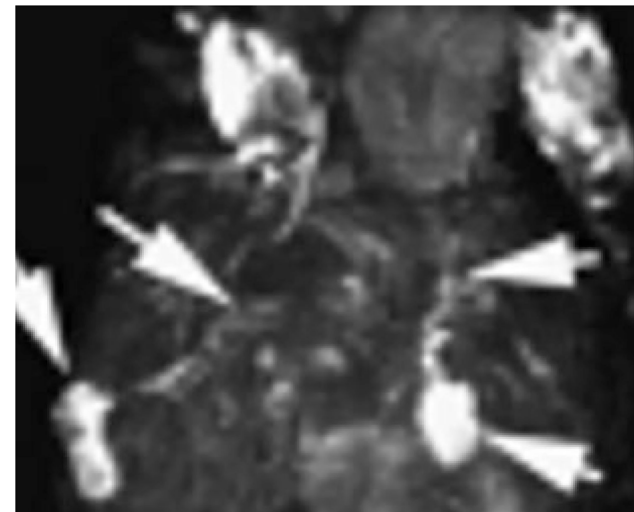

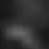

FIGURE 5 |.

Whole body three dimensional MR lymphangiogram (maximal intensity projection) of a normal mouse. PAMAM-G8 was injected intracutaneously into middle phalanges of all four limbs. Arrows indicate enhanced lymph nodes and lymphatic vessels. (Adapted with permission from Ref 70. Copyright 2006 Wiley Periodicals Inc). 


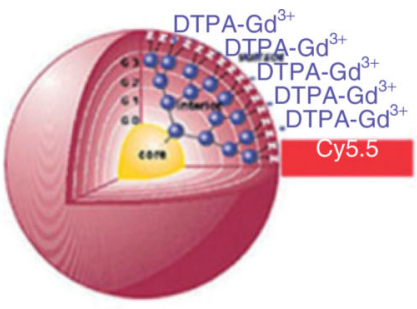

MRI \& NIR florescence hybrid nanoprobe G6-(DTPA-Gd) ${ }_{172}$-Cy5.5
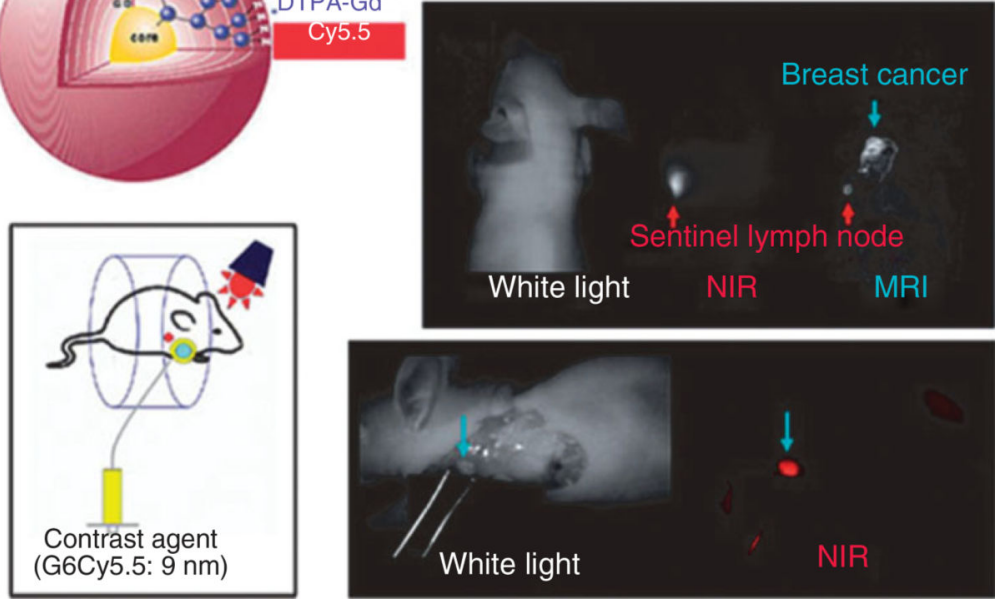

FIGURE 6 |.

Magnetic resonance imaging (MRI) and near-infrared (NIR) optical images of a sentinel lymph node of breast cancer in a mouse obtained with a MRI-NIR optical hybrid nano-sized contrast agent G6-Cy5.5 (left upper). Invivo (right upper) and intra-operative (right lower) images are shown along with a schematic illustration of the imaging method (left lower). (Adapted with permission from Ref 70. Copyright 2006 Wiley Periodicals Inc). 

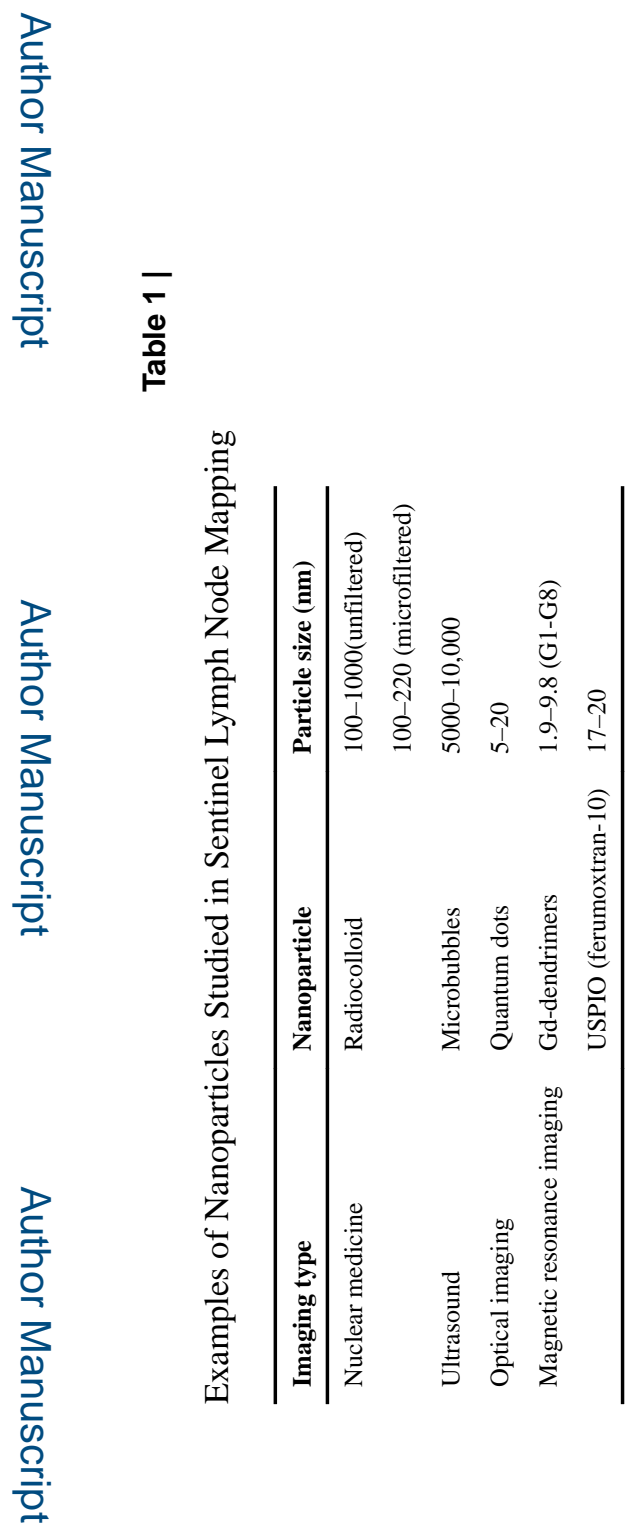

Wiley Interdiscip Rev Nanomed Nanobiotechnol. Author manuscript; available in PMC 2018 December 28. 